\title{
Approximation Algorithms for 3D Orthogonal Knapsack
}

\author{
Florian Diedrich ${ }^{1}$, Rolf Harren ${ }^{2}$, Klaus Jansen ${ }^{1}$, Ralf Thöle ${ }^{1}$, and Henning Thomas ${ }^{3}$ \\ ${ }^{1}$ Institute of Computer Science, University of Kiel, 24098 Kiel, Germany \\ ${ }^{2}$ Max-Planck-Institut für Informatik, Campus E1 4, 66123 Saarbrücken, Germany \\ ${ }^{3}$ Department of Computer Science, ETH Zurich, Zurich, Switzerland \\ E-mail: \{fdi, kj, rth\}@informatik.uni-kiel.de; Rolf.Harren@uni-dortmund.de; hthomas@inf.ethz.ch \\ Revised July 10, 2008.
}

\begin{abstract}
We study non-overlapping axis-parallel packings of 3D boxes with profits into a dedicated bigger box where rotation is either forbidden or permitted, and we wish to maximize the total profit. Since this optimization problem is NP-hard, we focus on approximation algorithms. We obtain fast and simple algorithms for the non-rotational scenario with approximation ratios $9+\epsilon$ and $8+\epsilon$, as well as an algorithm with approximation ratio $7+\epsilon$ that uses more sophisticated techniques; these are the smallest approximation ratios known for this problem. Furthermore, we show how the used techniques can be adapted to the case where rotation by $90^{\circ}$ either around the $z$-axis or around all axes is permitted, where we obtain algorithms with approximation ratios $6+\epsilon$ and $5+\epsilon$, respectively. Finally our methods yield a 3D generalization of a packability criterion and a strip packing algorithm with absolute approximation ratio 29/4, improving the previously best known result of $45 / 4$.
\end{abstract}

Keywords approximation algorithm, computational and structural complexity, geometric configurations

\section{Introduction}

Given a list $L=\left\{R_{1}, \ldots, R_{n}\right\}$ of boxes with sizes $R_{i}=\left(x_{i}, y_{i}, z_{i}\right)$ and positive profits $p_{i}$ for each $i \in$ $\{1, \ldots, n\}$ and a dedicated bigger box $Q=(a, b, c)$, we study non-overlapping axis-parallel packings of sublists of $L$ into $Q$ which we call feasible. For simplicity we call $Q$ a bin. We wish to select a sublist which permits a packing and maximizes the profit. This problem will be called the orthogonal three-dimensional knapsack problem or OKP-3, for short, and we denote the optimal profit by OPT. It is a natural generalization of the knapsack problem (KP) which is known to be NPhard. This makes an exact algorithm with a polynomial worst-case runtime bound impossible unless $\mathrm{P}=\mathrm{NP}$ holds. For this reason, we concentrate on approximation algorithms; we refer the reader to [1] for a detailed description of the approach and common notions. W.l.o.g., we assume $a=b=c=1$ and that each $R_{i} \in L$ can be packed by otherwise removing infeasible boxes and scaling in $\mathcal{O}(n)$ time. Note that the scaling is only possible in the non-rotational case. In the rotational case the fixed size of the bin is an explicit assumption.
Related Problems. Different geometrically-constrained two- and three-dimensional packing problems were studied, resulting in three main directions.

In strip packing, the target area is a strip of infinite height; the objective is to minimize the height of the packing. For the $2 \mathrm{D}$ case, Sleator ${ }^{[2]}$ proved an approximation ratio of $5 / 2$; Baker et al. ${ }^{[3]}$ obtained an asymptotic approximation ratio of $5 / 4$. The best known absolute approximation ratio of 2 was obtained independently with different techniques by Schiermeyer ${ }^{[4]}$ and Steinberg ${ }^{[5]}$. Kenyon and Rémila ${ }^{[6]}$ found an AFPTAS (asymptotic fully polynomial time approximation scheme) for the problem, and they obtained an additive error of $\mathcal{O}\left(1 / \epsilon^{2}\right)$. This additive error was later improved by Jansen and Solis-Oba in [7], where the authors presented an APTAS (asymptotic polynomial time approximation scheme) with additive error 1 . For the 3D case, research has focused mainly on the asymptotic approximation ratio, where Miyazawa and Wakabayashi ${ }^{[8]}$ found an algorithm with asymptotic approximation ratio of $27 / 10$ at most. An asymptotic ratio of $2+\epsilon$ was obtained by Jansen and Solis-Oba ${ }^{[9]}$; this was improved to 1.691 by Bansal et al. ${ }^{[10]}$ The best known absolute

Regular Paper

Research supported in part by DFG Project, Entwicklung und Analyse von Approximativen Algorithmen für Gemischte und Verallgemeinerte Packungs-und Überdeckungsprobleme, JA 612/10-1, in part by the German Academic Exchange Service DAAD, in part by project AEOLUS, under EU Contract No. 015964, and in part by a Grant "DAAD Doktorandenstipendium" of the German Academic Exchange Service DAAD. Part of this work was done in duration of visit to the LIG, Grenoble University. 
approximation ratio of $45 / 4$ follows from an asymptotic approximation ratio of $13 / 4$ from [11]. Li and Cheng ${ }^{[12]}$ studied the on-line version, resulting in a competitive ratio of $29 / 10$.

In bin packing, the objective is to minimize the number of identical bins. For the 1D case, an APTAS was presented by Fernandez de la Vega and Lueker ${ }^{[13]}$, while $\mathrm{Li}$ and $\mathrm{Yue}^{[14]}$ proved the bound of $11 / 9 \mathrm{OPT}+7 / 9$ for the popular FFD (first-fit decreasing height) algorithm. The study of FFD was settled recently by Dósa $^{[15]}$ who proved that the bound $11 / 9 \mathrm{OPT}+6 / 9$ is tight. For the $2 \mathrm{D}$ case, an asymptotic approximation ratio of 1.691 was obtained by Caprara ${ }^{[16]}$. This result was improved to an asymptotic approximation ratio of 1.525 by Bansal et al. ${ }^{[17]}$ Furthermore, Bansal et al. ${ }^{[18]}$ proved that $2 \mathrm{D}$ bin packing does not admit an APTAS (asymptotic polynomial time approximation scheme), and therefore there is no FPTAS (fully polynomial time approximation scheme) if $\mathrm{P} \neq \mathrm{NP}$. They also presented an APTAS for packing $d$-dimensional cubes into the minimum number of unit cubes in the same paper.

In knapsack scenario, the number of bins is a fixed constant $^{[19]}$, usually 1 . For the $2 \mathrm{D}$ case, Jansen and Zhang $^{[20]}$ obtained an approximation ratio of $2+\epsilon$. Classical 1D knapsack problems are relatively well understood, see [21], [22] for surveys.

Although these problems are closely related, the results cannot be transferred directly. One main contrast between bin and strip packing on the one hand and knapsack on the other hand is that in the first setting all boxes of the instance must be packed but in the latter a feasible selection of items must be found.

Previous Results and Applications. Harren ${ }^{[23]}$ obtained a ratio of $9 / 8+\epsilon$ for the special case of packing cubes, and Chlebík and Chlebíková proved the APXcompleteness of the general case ${ }^{[24]}$.

A cutting stock application is cutting blocks with given profits from larger pieces of material to maximize the profit. Another application is the problem of selecting boxes to be transported in a container. Besides these, the problem is motivated from multiprocessor scheduling on grid topology. In this perspective, for a time slice of fixed duration, a set of jobs to be executed must be chosen and each job requires a subgrid of prespecified rectangular shape. For a special case of this application, Ye and Zhang ${ }^{[25]}$ presented an on-line algorithm, see [26] for a study of similar problems.

New Results. Our contribution is a fast and simple $(9+\epsilon)$-approximation algorithm based on strip packing which is refined to an $(8+\epsilon)$-approximation algorithm. Both of these have practical running times. With more sophisticated techniques, we obtain a $(7+\epsilon)$-approxi- mation algorithm. We also study the case where rotation by $90^{\circ}$ either around the $z$-axis or around all axes is permitted, where we significantly improve upon the approximation ratios of the algorithms presented for OKP-3. We derived approximation ratios of $6+\epsilon$ for the former and $5+\epsilon$ for the latter case. Finally our methods yield a three-dimensional generalization of a packability criterion and a strip packing algorithm with absolute approximation ratio 29/4.

This paper is organized as follows. In Section 2, we present a fast algorithm for non-rotational packing which is improved in Section 3. In Section 4 we generalize a two-dimensional packability criterion for boxes, and improve a known result on strip packing in Section 5 before turning back to the knapsack problem in Section 6, where we obtain a better yet more costly algorithm. Finally, we discuss the cases of rotational packing in Section 7, and conclude the paper with open problems in Section 8.

\section{Algorithm Based on Strip Packing}

We approximately solve a relaxation by selecting $L^{\prime} \subseteq L$ that is at least near-optimal and has a total volume of at most 1 . This relaxed solution is partitioned into 9 sublists. For each of these a packing into the bin will be generated. Out of these, one with the maximum profit is chosen, resulting in a $(9+\epsilon)$-approximation algorithm. More precisely $L^{\prime}$ will be packed into a strip $[0,1] \times[0,1] \times[0, \infty)$ by a level-oriented algorithm, i.e., an algorithm which packs all boxes into disjoint levels and stacks these levels on top of one another into the strip. We partition the strip into packings of sublists of $L^{\prime}$ and among these return one with the maximum profit.

For each box $R_{i}$, the rectangle $\left(x_{i}, y_{i}\right)$ is called the base rectangle of $R_{i}$, denoted as $b r\left(R_{i}\right)$. Such a rectangle $\left(x_{i}, y_{i}\right)$ is called

$$
\begin{aligned}
& b i g: \Leftrightarrow x_{i} \in(1 / 2,1] \quad \text { and } \quad y_{i} \in(1 / 2,1], \\
& \text { long }: \Leftrightarrow x_{i} \in(1 / 2,1] \text { and } y_{i} \in(0,1 / 2] \text {, } \\
& \text { wide }: \Leftrightarrow x_{i} \in(0,1 / 2] \text { and } y_{i} \in(1 / 2,1] \text {, } \\
& \text { small }: \Leftrightarrow x_{i} \in(0,1 / 2] \quad \text { and } \quad y_{i} \in(0,1 / 2] \text {. }
\end{aligned}
$$

For each list $L$ of boxes we use $V(L)$ to denote the total volume of $L$, and for each list $\hat{L}$ of rectangles use $A(\hat{L})$ to denote the total area of $\hat{L}$. Furthermore, $P(L)$ denotes the total profit of $L$. Finally, for each list $L$ of boxes we use $H(L)$ to denote the height of a packing of $L$ where the packing itself will be clear from the context. We use the following theorem from [20] which is a refinement of the main result from [5]. 
Theorem $\mathbf{1}^{[\mathbf{2 0}]}$. Let $L$ be a list of $n$ rectangles such that $A(L) \leqslant 1 / 2$ holds, and either no long or no wide rectangles occur in $L$, i.e., there might be either long or wide rectangles, but not both. Then $L$ permits a feasible packing into the unit square which can be generated in time $\mathcal{O}\left(n \log ^{2} n / \log \log n\right)$.

Note that rectangles which are long or wide are not big. It is therefore possible in Theorem 1 that there is a big rectangle in $L$. First we apply the modified strip packing algorithm, and then we construct the partition of the strip. The strip packing algorithm uses Theorem 1 to obtain an area guarantee for each level but the last, improving a result from [11].

\section{Algorithm A}

1. Partition $L$ into $L_{1}:=\left\{R_{i} \mid \operatorname{br}\left(R_{i}\right)\right.$ is long $\}$ and $L_{2}:=L \backslash L_{1}$. W.l.o.g., we have

$$
L_{1}=\left\{R_{1}, \ldots, R_{m}\right\}
$$

and

$$
L_{2}=\left\{R_{m+1}, \ldots, R_{n}\right\} .
$$

2. Generate the packing for $L_{1}$ as follows.

2.1. Find the boxes $R_{i}$ in $L_{1}$ for which the area of $b r\left(R_{i}\right)$ is greater than $1 / 4$, which are $R_{p+1}, \ldots, R_{m}$, w.l.o.g., Stack these on top of one another in direction $z$, each on its own level.

2.2. Sort the remaining boxes $R_{1}, \ldots, R_{p}$ in nonincreasing order of $z_{i}$, resulting in a list $L_{1}^{\prime}$.

2.3. Partition $L_{1}^{\prime}$ into consecutive sublists $L_{1}^{\prime \prime}, \ldots, L_{v}^{\prime \prime}$ where the total base area of each sublist is as close to $1 / 2$ as possible but not greater. Pack each of these sublists on a level by itself using Theorem 1. Stack all of these levels on top of one another in direction $z$.

3. Generate the packing for $L_{2}$ in a similar way as for $L_{1}$ by Theorem 1 . The resulting steps are called Steps $3.1 \sim 3.3$.

4. Concatenate the packings of $L_{1}$ and $L_{2}$ to obtain a packing of $L$.

Theorem 2. For each list $L$ of $n$ boxes, Algorithm $A$ generates a packing of height at most $4 V(L)+Z_{1}+Z_{2}$, where $Z_{1}$ and $Z_{2}$ are the heights of the first levels generated in Steps 2.3 and 3.3. The construction can be carried out in time $\mathcal{O}\left(n \log ^{2} n / \log \log n\right)$.

Proof. Consider Step 2.3 which generates levels $L_{1}^{\prime \prime}, \ldots, L_{v}^{\prime \prime}$. Let $h_{i}$ denote the height of level $L_{i}^{\prime \prime}$ for each $i \in\{1, \ldots, v\}$. Each box $R_{i}$ for $i \in\{p+1, \ldots, m\}$ processed in Step 2.1 forms a level itself, so the total height for the packing of $L_{1}$ is

$$
H\left(L_{1}\right):=\sum_{i=p+1}^{m} z_{i}+\sum_{i=1}^{v} h_{i},
$$

and we have $V(L)=V\left(L_{1}\right)+V\left(L_{2}\right)$. Furthermore,

$$
V\left(L_{1}\right)=\sum_{i=p+1}^{m} x_{i} y_{i} z_{i}+\sum_{i=1}^{v} V\left(L_{i}^{\prime \prime}\right)
$$

holds. In Step 2.1 we assert that $x_{i} y_{i}>1 / 4$ for $i \in\{p+1, \ldots, m\}$, so we get

$$
\sum_{i=p+1}^{m} x_{i} y_{i} z_{i}>\frac{1}{4} \sum_{i=p+1}^{m} z_{i} .
$$

For each $i \in\{1, \ldots, v-1\}$, the total base area of the boxes in $L_{i}^{\prime \prime}$ is larger than $1 / 4$ since otherwise the next box would have also been put in $L_{i}^{\prime}$ in Step 2.3. As the boxes are sorted in non-increasing order of height, and thus $z_{j} \geqslant h_{i+1}$ for $R_{j} \in L_{i}^{\prime \prime}$, we get that

$$
\sum_{R_{j} \in L_{i}^{\prime \prime}} x_{j} y_{j}>\frac{1}{4}
$$

holds, hence

$$
V\left(L_{i}^{\prime \prime}\right) \geqslant \frac{1}{4} h_{i+1}
$$

for each $i \in\{1, \ldots, v-1\}$. Combining (2) and (4) results in

$$
V\left(L_{1}\right)>\frac{1}{4} \sum_{i=p+1}^{m} z_{i}+\frac{1}{4} \sum_{i=2}^{v} h_{i}
$$

which together with (1) implies that

$$
H\left(L_{1}\right)<4 V\left(L_{1}\right)+h_{1} \leqslant 4 V\left(L_{1}\right)+Z_{1}
$$

holds. For Step 3 we get

$$
H\left(L_{2}\right)<4 V\left(L_{2}\right)+Z_{2}
$$

with a very similar analysis. From (5) and (6) we conclude that the height of our packing of $L$ is bounded from above by

$$
H\left(L_{1}\right)+H\left(L_{2}\right)<4 V(L)+Z_{1}+Z_{2} .
$$

The runtime is dominated by the application of Theorem 1 and thus bounded by $\mathcal{O}\left(n \log ^{2} n / \log \log n\right)$.

The second part of the algorithm is a partition of the arrangement generated by Algorithm A into at most 9 bins, see Fig.1.

\section{Algorithm B}

1. Set $\delta:=\epsilon /(9+\epsilon)$. Use an FPTAS for $\mathrm{KP}$ from $[21,27]$ to select $L^{\prime} \subseteq L$ such that $V\left(L^{\prime}\right) \leqslant 1$ and $P\left(L^{\prime}\right) \geqslant(1-\delta) O P T$ holds, where OPT denotes the optimum of the generated KP instance.

2. Use Algorithm A to generate a packing of $L^{\prime}$ into the strip but separate the first levels generated in Steps 2.3 and 3.3. Pack these into a bin each. 
3. By Theorem 2 the remaining strip has a height of at most $4 V\left(L^{\prime}\right) \leqslant 4$. Consider the cutting unit squares $[0,1] \times[0,1] \times\{i\}$ for $i \in\{1,2,3\}$. Generate a partition of the region $[0,1] \times[0,1] \times[0,4]$ into 7 subsets, i.e., 4 subsets which are positioned each in the regions $[0,1] \times[0,1] \times[i-1, i]$ for $i \in\{1, \ldots, 4\}$ but not intersecting any of the unit squares, and 3 subsets of boxes each of which intersects with one of the three cutting unit squares.

4. Out of the sets generated in Steps 2 and 3 returns one with the maximum profit.
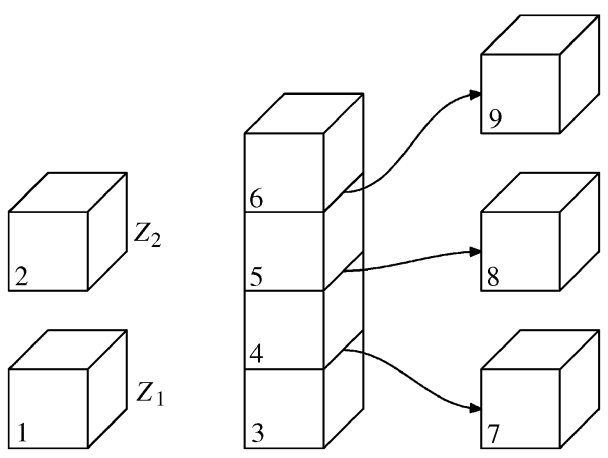

Fig.1. At most 9 bins are generated by Algorithm B.

Each set generated in Steps 2 and 3 permits a feasible packing into the unit cube which is available as a byproduct of Algorithm A. $L^{\prime}$ is partitioned into at most 9 subsets by Algorithm B, as illustrated in Fig.1.

Theorem 3. Algorithm $B$ is a $(9+\epsilon)$-approximation algorithm for OKP-3 with running time $\mathcal{O}\left(T_{\mathrm{KP}}(n, \epsilon)+n \log ^{2} n / \log \log n\right)$, where $T_{\mathrm{KP}}(n, \epsilon)$ is the running time of the FPTAS used for KP from $[21,27]$; furthermore, this bound is tight. Note that in [21] the best running time for an FPTAS for the knapsack problem is

$$
\begin{aligned}
\mathcal{O}(n \min \{ & \log n, \log (1 / \epsilon)\} \\
& \left.+1 / \epsilon^{2} \log (1 / \epsilon) \min \{n, 1 / \epsilon \log (1 / \epsilon)\}\right) .
\end{aligned}
$$

Proof. Clearly $9+\epsilon$ is an upper bound for the ratio and the running time is dominated by solving the knapsack instance and by Algorithm A since

$$
\frac{(1-\delta)}{9}=\frac{1}{9+\epsilon}
$$

holds. For the following instance this bound can be attained. We have 10 boxes $R_{1}:=(1 / 2,1 / 2,2 / 15)$, $R_{2}:=(1,1 / 4,2 / 15), \quad R_{3}:=(1,2 / 7,3 / 4), \quad R_{4}:=$ $\cdots:=R_{7}:=(1,2 / 7,1 / 2), R_{8}:=(1,2 / 7,1 / 4+2 / 15)$, $R_{9}:=(1,2 / 7,2 / 15)$ and $R_{10}:=(1,1,1)$. Furthermore, $p_{1}:=\cdots:=p_{9}:=1 /(9+\epsilon)$, and $p_{10}:=1$. Let $S_{1}:=\left\{R_{1}, \cdots, R_{9}\right\}$ and $S_{2}:=\left\{R_{10}\right\}$. It is clear that
$S_{2}$ is an optimal solution. Elementary calculation shows $V\left(S_{1}\right)=1$ and $P\left(S_{1}\right)=1-\delta$, and hence $S_{1}$ may be selected in Step 1 of Algorithm B. Application of Algorithm $\mathrm{B}$, assuming that the boxes are stacked in increasing order of index in Step 2.1 of Algorithm A, yields 9 bins each containing an item with profit $1 /(9+\epsilon)$, see Fig.2.
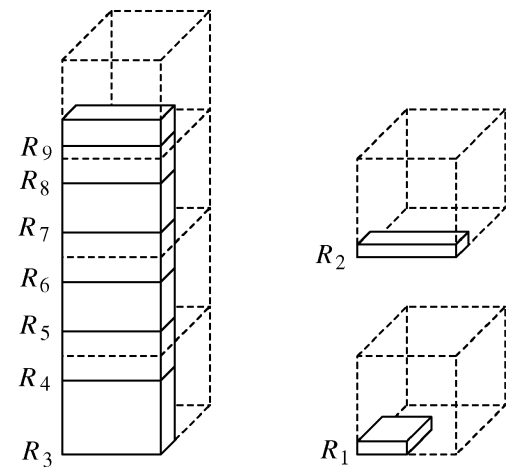

Fig.2. Packing of $S_{1}$ with Algorithm B.

Note that only the subset that is returned needs to be packed level-wise using the algorithm from Theorem 1 while the discarded subsets need not be arranged. Algorithm B can be used to solve the special cases where we wish to maximize the number of selected boxes or the volume by setting $p_{i}:=1$ or $p_{i}:=x_{i} y_{i} z_{i}$ for each $i \in\{1, \ldots, n\}$. This also holds for the other algorithms which we present.

In [21, 27] approximation algorithms for various knapsack problems are found. Using them, Algorithm B can be generalized by replacing the KP solver in Step 1, yielding algorithms for unbounded OKP-3 and multiple-choice OKP-3, see [21, 27] for notions and details. Algorithm B can be modified to yield a ratio of 18 with a much better running time by using a 2approximation algorithm for classical KP from [21, 27], thus replacing $T_{\mathrm{KP}}(n, \epsilon)$ by $\mathcal{O}(n)$ in Theorem 3 .

\section{Refined Construction}

In this section we show how to refine Algorithm B to yield an approximation ratio of $(8+\epsilon)$. We identify two possible improvements on Algorithm A: first, by increasing the area guarantee and second, by decreasing the heights $Z_{1}$ and $Z_{2}$ of the additional strip packing levels.

In Algorithm $\mathrm{A}$ and the proof of Theorem 2, the area bound $1 / 2$ from Theorem 1 was used. We separated boxes with base area greater than $1 / 4$, resulting in the area guarantee of $1 / 2-1 / 4=1 / 4$ for each level generated in Steps 2.2 and 2.3 except the last one. By 
improving the area guarantee we will improve the height bound of the strip.

So far we arbitrarily chose direction $z$ to be the axis for level generation, but any direction $d \in\{x, y, z\}$ will do. This has the advantage that packing in a direction where all boxes are short, i.e., at most $1 / 2$, implies that the heights of the additional levels are bound by $1 / 2$.

Let us introduce the notion of big boxes in certain directions: for any direction $d \in\{x, y, z\}$ a box $R_{i}$ is called $d$-big: $\Leftrightarrow d_{i} \in(1 / 2,1]$, and we use $X, Y$ and $Z$ to denote the set of boxes that are $d$-big for the corresponding direction. Any box that is $d$-big for every direction $d \in\{x, y, z\}$ will be called a big box. Finally, a box $R_{i}$ is called small $: \Leftrightarrow x_{i} \in(0,1 / 2], y_{i} \in(0,1 / 2]$, and $z_{i} \in(0,1 / 2]$.

The remainder of the section is organized as follows. First, we give a refined version of Algorithm A that is restricted to packing small boxes. Second, we show how to partition the boxes which are not small into three sets according to the big direction. Third, we give the overall algorithm which is based on the partition into $d$-big and small boxes.

The following algorithm is applied only on small items.

\section{Algorithm C}

1. Find the boxes $R_{i}$ in $L$ for which the area of $\operatorname{br}\left(R_{i}\right)$ is greater than $1 / 10$, and they are $R_{1}, \ldots, R_{m}$, w.l.o.g., Sort these in non-increasing order of $z_{i}$, resulting in a list $L_{1}$. Arrange these in groups of 4 boxes each, except for the last group. Each group can be put on a separate level by placing the boxes into the corners of the level. Stack these levels on top of one another in direction $z$.

2. Sort the remaining boxes $R_{m+1}, \ldots, R_{n}$ in nonincreasing order of $z_{i}$, resulting in a list $L_{2}$.

3. Partition $L_{2}$ into consecutive sublists $L_{1}^{\prime \prime}, \ldots, L_{v}^{\prime \prime}$ where the total base area of each sublist is as close to $1 / 2$ as possible but not greater. Pack each of these sublists on a level by itself using Theorem 1. Stack all of these levels on top of one another in direction $z$.

4. Concatenate the packings of $L_{1}$ and $L_{2}$ to obtain a packing of $L$.

Note that we formed two groups and obtained an area guarantee of $2 / 5=1 / 2-1 / 10$ for each layer except the last ones generated in Steps 1 and 3. To avoid confusion we point out that the area guarantee does not hold for the last generated layers, while the summands $Z_{1}$ and $Z_{2}$ in Theorem 2 are the heights of the respective first layers. Similarly to the proof of Theorem 2, we obtained the following results using the area guarantee of $2 / 5$.

Theorem 4. For each list $L$ of $n$ small boxes, Algorithm $C$ generates a feasible packing of height at most

$$
5 / 2 V(L)+Z_{1}+Z_{2},
$$

where $Z_{1} \leqslant 1 / 2$ and $Z_{2} \leqslant 1 / 2$ are the heights of the first levels generated in Steps 1 and 3 . The construction can be carried out in time $\mathcal{O}\left(n \log ^{2} n / \log \log n\right)$.

Lemma 5. Each list $L$ of $n$ small boxes with $V(L) \leqslant 1$ permits a feasible packing into at most 5 bins. The construction can be carried out in time $\mathcal{O}\left(n \log ^{2} n / \log \log n\right) ;$ the bound of 5 is tight for the used construction.

Proof. Use Algorithm $\mathrm{C}$ to arrange $L$ in a strip, but separate the first levels generated in Steps 1 and 3 . Since $L$ contains only small boxes, these two levels can be packed together into a bin. By Theorem 4, the remaining strip has a height of at most $5 / 2$. Consider the two cutting unit squares $[0,1] \times[0,1] \times\{i\}$ for $i \in\{1,2\}$. Generate a partition of the region $[0,1] \times[0,1] \times[0,5 / 2]$ into 5 subsets, namely first 3 subsets which are each positioned in the regions $[0,1] \times[0,1] \times[i-1, i]$ for $i \in\{1,2\}$ as well as the region $[0,1] \times[0,1] \times[2,5 / 2]$ but not intersecting any of the unit squares, and furthermore the rest 2 subsets of boxes each of which intersects with one of the two cutting unit squares. The first three sets can be packed into one bin each. Since $L$ contains only small boxes, the last two sets can be arranged together into one additional bin by aligning them at the top and bottom of the bin, respectively. We have at most 5 bins, see Fig.3. The running time is dominated by Algorithm $\mathrm{C}$ and thus bounded by $\mathcal{O}\left(n \log ^{2} n / \log \log n\right)$.
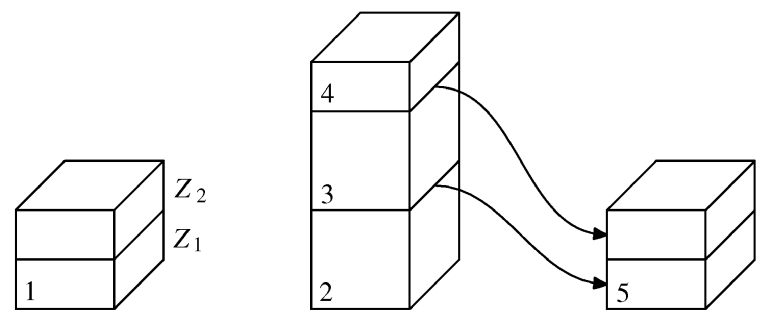

Fig.3. Small boxes can be packed into at most 5 bins.

To show the tightness of the bound, let $\gamma:=1 / 500$ and consider the instance $L$ consisting of $R_{1}:=\cdots:=$ $R_{29}:=(1 / 2,1 / 5+\gamma, 1 / 3+\gamma), R_{30}:=(\gamma, \gamma, 1 / 2)$, $R_{31}:=\cdots:=R_{33}:=(1 / 2,1 / 5, \gamma)$ and $R_{34}:=$ $\left(1 / 2,1 / 5-2 \gamma^{2}, \gamma\right)$. Note that

$$
\begin{aligned}
V(L) & =29 / 30+122 / 15 \gamma+15 \gamma^{2}-\gamma^{3} \\
& <29 / 30+1 / 60
\end{aligned}
$$

Application of Algorithm C packs $R_{1}, \ldots, R_{29}$ in Step 1 , resulting in 8 layers. All remaining boxes are packed into one more layer in Step 3. The height of each layer 
is greater than $1 / 3$, which means that the layers cannot be arranged in less than 5 bins.

A partition of the boxes which are not small is given by the following lemma. In the sequel we use the notion of the projection of a box; for each box $R=(x, y, z)$ we call the rectangle $(y, z)$ the $x$-projection, the rectangle $(x, z)$ the $y$-projection, and the rectangle $(x, y)$ the $z$ projection of $R$.

Lemma 6. Let $L$ be a list of $n$ boxes in which no small boxes and at most 3 big boxes occur. Then $L$ can be partitioned into sets $X^{\prime}, Y^{\prime}$ and $Z^{\prime}$ in time $\mathcal{O}(n)$, such that each of these contains at most one big box, and the $x$-projections of boxes in $X^{\prime}$, the $y$-projections of boxes in $Y^{\prime}$ and the $z$-projections of boxes in $Z^{\prime}$ contain no long or no wide rectangles.

Proof. Remove the at most 3 big boxes from $L$ and distribute them in $X^{\prime}, Y^{\prime}$ and $Z^{\prime}$ such that in each of these sets at most one big box occurs. Set $X^{\prime}:=X^{\prime} \cup\left\{R_{i} \in L \mid x_{i}>1 / 2, z_{i} \leqslant 1 / 2\right\}, Y^{\prime}:=$ $Y^{\prime} \cup\left\{R_{i} \in L \mid y_{i}>1 / 2, x_{i} \leqslant 1 / 2\right\}$ and finally $Z^{\prime}:=$ $Z^{\prime} \cup\left\{R_{i} \in L \mid z_{i}>1 / 2, y_{i} \leqslant 1 / 2\right\}$ to obtain the claim. To see that $X^{\prime}, Y^{\prime}$ and $Z^{\prime}$ form a partition, consider w.l.o.g., a box $R$ that is $x$-big. If this box is $y$-big and $z$-big, it is big and therefore included in one of the sets. Otherwise it is either $z$-big and in $Z^{\prime}$ or small in direction $z$ and thus in $X^{\prime}$. On the other hand, no box can be in more than one of the sets.

We are now ready to give the overall algorithm. To avoid repetition, we enumerate the cases in the analysis only.

\section{Algorithm D}

1. Set $\delta:=\epsilon /(8+\epsilon)$. Use a PTAS for non-geometric $4 \mathrm{D} \mathrm{KP}$ from $[21,27]$ to select $L^{\prime} \subseteq L$ such that $P\left(L^{\prime}\right) \geqslant(1-\delta) O P T$, where OPT, denotes the optimum of the integral linear program

$$
\text { maximize } \sum_{i=1}^{n} p_{i} R_{i} \quad \text { subject to } \quad R \in P,
$$

where $R_{i}$ is an indicator variable for the box of the same name. Furthermore we define the set $P$ by the constraints

$$
\begin{aligned}
& \sum_{i=1}^{n} x_{i} y_{i} z_{i} R_{i} \leqslant 1 \\
& \sum_{R_{i} \in X} y_{i} z_{i} R_{i} \leqslant 1 \\
& \sum_{R_{i} \in Y} x_{i} z_{i} R_{i} \leqslant 1, \\
& \sum_{R_{i} \in Z} x_{i} y_{i} R_{i} \leqslant 1 .
\end{aligned}
$$

In total, $P$ is a polytope of nonnegative integers.
2. Partition $L^{\prime}$ into at most 8 subsets which permit a feasible packing as described below. Out of these, returns one with the maximum profit.

Theorem 7. Algorithm $D$ is a $(8+\epsilon)$-approximation algorithm for OKP-3 with running time $\mathcal{O}\left(\mathrm{T}_{4 \mathrm{DKP}}(n, \epsilon)+n \log ^{2} n / \log \log n\right)$, where $T_{4 \mathrm{DKP}}(n, \epsilon)$ is the running time of the PTAS used for $4 D K P$ from $[21,27]$; furthermore this bound is tight. Note that in [21] the best running time for a PTAS for the multidimensional knapsack problem is $\mathcal{O}\left(n^{\lceil d / \epsilon\rceil-d}\right)$.

Proof. The first constraint of the integral linear program models the volume bound of the box. The other constraints are area bounds for $d$-big boxes for $d \in\{x, y, z\}$, motivated by the observation that the $d$ projections of $d$-big boxes do not overlap. Thus the given program is a relaxation of our problem.

We have not imposed a bound on the number of big boxes in the relaxation, but due to the area conditions there are at most $3 \mathrm{big}$ boxes in the selected set. We consider two cases according to the total projection area of the $d$-big boxes.

Case 1. There is a direction $d \in\{x, y, z\}$ such that the total $d$-projection area of all $d$-big boxes in $L^{\prime}$ is larger than or equal to $1 / 2$. In this case all $d$-big boxes can be packed into at most 3 bins with a construction from [20], which can be done in time $\mathcal{O}\left(n \log ^{2} n / \log \log n\right)$, resulting in a volume of at least $1 / 4$ being packed. The total volume of the remaining boxes is bounded by $3 / 4$ and each remaining box has a $d$-height of at most $1 / 2$. We apply Algorithm $\mathrm{A}$ in direction $d$ which results in a strip of $d$-height at most 3 and two additional levels of $d$-height at most $1 / 2$ each. Similar to the proof of Lemma 5 all these sets can be packed into at most 5 bins, see Fig.3, generating at most 8 bins in total.

Case 2. For each $d \in\{x, y, z\}$ the total projection area of all $d$-big boxes is smaller than $1 / 2$. By Lemma 6 we partition the set $\left\{R_{i} \in L^{\prime} \mid R_{i}\right.$ is not small $\}$ into sets $X^{\prime}, Y^{\prime}$ and $Z^{\prime}$ such that the total projection area of $X^{\prime}, Y^{\prime}$ and $Z^{\prime}$ for the corresponding direction is not greater than $1 / 2$, and the $x$-projections of boxes in $X^{\prime}$, the $y$-projections of boxes in $Y^{\prime}$ and the $z$-projection of boxes in $Z^{\prime}$ contain no long or no wide rectangles, respectively. Furthermore, each of these sets contains at most one big box. By Theorem 1 the sets $X^{\prime}, Y^{\prime}$ and $Z^{\prime}$ can be packed into at most one bin each, resulting in at most 3 bins in total. Let $S$ denote the set of small boxes; these are not yet packed. Clearly $V(S) \leqslant 1$ holds, so by Lemma 5 the set $S$ can be packed into at most 5 bins, which results in at most 8 bins in total. The runtime bound follows from the fact that we can distinguish between the two cases in time $\mathcal{O}(n)$. 
For the tightness of the bound, consider the instance $L$ in which $R_{1}, \ldots, R_{34}$ are as in the proof of Lemma 5, $R_{35}:=(1,1,1 / 180), R_{36}:=(1,1 / 180,1), R_{37}:=$ $(1 / 180,1,1)$, and $R_{38}:=(1,1,1)$. The profits are defined by $p_{i}:=1 /[9(8+\epsilon)]$ for $i \in\{1, \ldots, 4,30, \ldots, 34\}$, $p_{i}:=1 /[8(8+\epsilon)]$ for $i \in\{5, \ldots, 28\}, p_{i}:=1 /(8+\epsilon)$ for $i \in\{29,35,36,37\}$, and $p_{38}:=1$. Let $S_{1}:=L \backslash\left\{R_{38}\right\}$ and $S_{2}:=\left\{R_{38}\right\}$. Since $P\left(S_{1}\right)=8 /(8+\epsilon)=(1-\delta)<$ $1=P\left(S_{2}\right), S_{2}$ is an optimal solution. Elementary calculation verifies that $S_{1}$ may be chosen in Step 1 of Algorithm D. Application of Algorithm D leads to Case 2 in the analysis above, where $X^{\prime}=\left\{R_{35}\right\}, Y^{\prime}=\left\{R_{37}\right\}$ and $Z^{\prime}=\left\{R_{36}\right\}$. Each of these sets is packed into a separate bin. The remaining boxes are small and are packed into 5 bins as in the proof of Lemma 5. In total, 8 bins are generated; the profits are chosen such that each bin yields a profit of exactly $1 /(8+\epsilon)$.

\section{Packability Criterion}

Theorem 1 has a number of applications besides the use in this paper, see $[20,28]$. In this section we give a generalization to Theorem 1 for the three-dimensional case.

Lemma 8. Let $L$ be a list of $n$ boxes such that $V(L) \leqslant 1 / 8$ holds, and no $d_{1}$-big and no $d_{2}$-big boxes occur in $L$ for $d_{1}, d_{2} \in\{x, y, z\}$ and $d_{1} \neq d_{2}$. Then $L$ permits a feasible packing into the unit bin which can be generated in time $\mathcal{O}\left(n \log ^{2} n / \log \log n\right)$.

Observe that similarly to Theorem 1 we are allowed to have items that are $d$-big in a certain direction $d$ but not in any other direction. A difference between the original two-dimensional version and our generalization is that in the original version a single big item - which is neither long nor wide - was allowed, but is forbidden here.

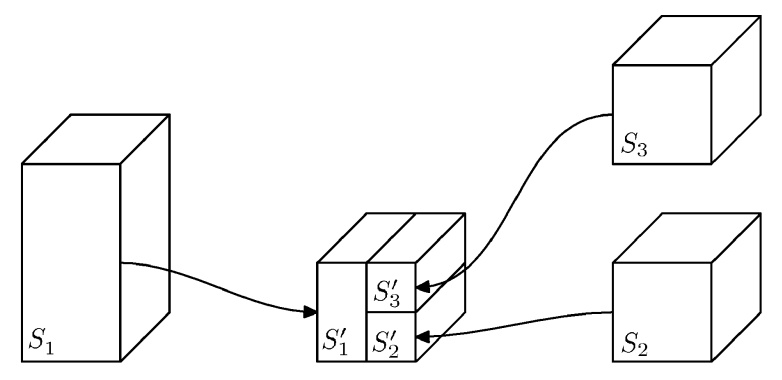

Fig.4. Arrangement of the stacks into a cube.

Proof. Assume w.l.o.g., assume that there are no $x$-big and no $z$-big boxes in $L$. Obtain the set $L_{\text {scaled }}$ by scaling the given instance by 2 in directions $x$ and $z$. We use Algorithm $\mathrm{A}$ in direction $z$ to pack the boxes in $L_{\text {scaled }}$ into one stack $S_{1}$ of height at most $4 V\left(L_{\text {scaled }}\right) \leqslant 4 \cdot 1 / 2=2$ and two additional stacks $S_{2}$ and $S_{3}$ of height limited by 1 which correspond to the summands $Z_{1}$ and $Z_{2}$ in Theorem 4 . Rescaling everything yields a stack $S_{1}^{\prime}$ of height at most 1 and two additional stacks $S_{2}^{\prime}$ and $S_{3}^{\prime}$ of heights $1 / 2$. All stacks have a width of at most $1 / 2$ in direction $x$. Thus we can arrange all stacks in a unit cube as shown in Fig.4.

\section{Improved Strip Packing Algorithm}

In Theorem 7 in [11], an approximation algorithm for $3 \mathrm{D}$ strip packing with asymptotic approximation ratio $13 / 4$ is presented, and more precisely the bound is $13 / 4 O P T+8 Z$, where $Z$ is the height of the highest item. In this section we show that the additive constant of this bound can be easily improved upon by using Theorem 1; more precisely we obtain the following result by using a more suitable subdivision which results in fewer groups.

Theorem 9. For each list $L$ of $n$ boxes a packing into the strip $[0,1] \times[0,1] \times[0, \infty)$ of height at most $13 / 4 O P T(L)+4 Z$ can be generated, where OPT denotes the minimum attainable packing height and $Z$ is the height of the highest box. The running time is polynomial in $n$.

As we already mentioned in the introduction, the currently best known asymptotic approximation ratio for this problem is 1.691 by Bansal et al. ${ }^{[10]}$ Our result improves the best known absolute approximation ratio of $45 / 4$ which follows from [11] to $29 / 4$.

Proof. The construction and the proof are similar as in [11] and included for completeness. We partition $L$ into five groups by letting

$$
\begin{aligned}
L_{1}:= & \left\{R_{i} \mid \operatorname{br}\left(R_{i}\right) \text { is big }\right\}, \\
L_{2}:= & \left\{R_{i} \mid \operatorname{br}\left(R_{i}\right) \text { is long and } A\left(\operatorname{br}\left(R_{i}\right)\right) \leqslant 1 / 6\right\}, \\
L_{3}:= & \left\{R_{i} \mid \operatorname{br}\left(R_{i}\right) \text { is long and } A\left(\operatorname{br}\left(R_{i}\right)\right)>1 / 6\right\}, \\
L_{4}:= & \left\{R_{i} \mid \operatorname{br}\left(R_{i}\right)\right. \text { is wide or small, } \\
& \text { and } \left.A\left(\operatorname{br}\left(R_{i}\right)\right) \leqslant 1 / 6\right\}, \text { and } \\
L_{5}:= & \left\{R_{i} \mid \operatorname{br}\left(R_{i}\right)\right. \text { is wide or small, } \\
& \text { and } \left.A\left(b r\left(R_{i}\right)\right)>1 / 6\right\},
\end{aligned}
$$

and discuss how to obtain good corresponding area guarantees. Clearly, an area guarantee of $1 / 4$ can be obtained for $L_{1}$ by simply stacking the boxes on top of one another. Furthermore, the groups $L_{2}, \ldots, L_{5}$ can be sorted in non-increasing order of $z_{i}$. Now for each of these, we proceed similarly as in Step 2.3 of Algorithm A. For $L_{2}$ we generate layers using Theorem 1 
and obtain an area guarantee of $1 / 2-1 / 6=1 / 3$ for each layer except the last one. In an even simpler way, the group $L_{3}$ can be packed by putting at least two boxes on each layer except for the last one; we obtain an area guarantee of $1 / 3$ for each but the last layer. By using Theorem 1, we generate layers for the items in $L_{4}$ and again obtain an area guarantee of $1 / 3$ for each layer but the last. Finally, the boxes of $L_{5}$ can be packed by placing two of them on each layer except for the last one; again we obtain an area guarantee of $1 / 3$ for each but the last layer.

Now let $H\left(L_{1}\right)$ denote the height of the packing generated for $L_{1}$ and, for each $i \in\{2, \ldots, 5\}$, let $H\left(L_{i}\right)$ denote the height of the packing of $L_{i}$ minus the height of the corresponding first layer generated. Let $H(L)$ denote the total height of the packing. For

$$
H:=\sum_{i=2}^{5} H\left(L_{i}\right)
$$

clearly

$$
H(L) \leqslant H\left(L_{1}\right)+H+4 Z
$$

holds. Furthermore, we have

$$
V\left(L_{1}\right) \geqslant \frac{H\left(L_{1}\right)}{4} .
$$

Now let $i \in\{2, \ldots, 5\}$ and let $L_{1}^{\prime}, \ldots, L_{j}^{\prime}$ denote the levels generated for $L_{i}$; finally let $V\left(L_{k}^{\prime}\right)$ denote the total volume of boxes in $L_{k}^{\prime}$ and let $h_{k}$ denote the height of $L_{k}^{\prime}$ for each $k \in\{1, \ldots, j\}$. Since for $L_{1}^{\prime}, \ldots, L_{j-1}^{\prime}$ we have an area guarantee of at least $1 / 3$, we obtain

$$
V\left(L_{i}\right)>\sum_{k=1}^{j-1} V\left(L_{k}^{\prime}\right) \geqslant \frac{1}{3} \sum_{k=2}^{j} h_{k}=\frac{H\left(L_{i}\right)}{3} .
$$

In total, we obtain

$$
O P T(L) \geqslant V(L)=\sum_{i=1}^{5} V\left(L_{i}\right)>\frac{H\left(L_{1}\right)}{4}+\frac{H}{3} .
$$

Obviously we also have $O P T(L) \geqslant H\left(L_{1}\right)$, and thus

$$
O P T(L) \geqslant \max \left\{H\left(L_{1}\right), \frac{H\left(L_{1}\right)}{4}+\frac{H}{3}\right\}
$$

holds. Now we study the ratio

$$
r:=\frac{H\left(L_{1}\right)+H}{\max \left\{H\left(L_{1}\right), \frac{H\left(L_{1}\right)}{4}+\frac{H}{3}\right\}} .
$$

If $H\left(L_{1}\right) \geqslant H\left(L_{1}\right) / 4+H / 3$, which means that $H\left(L_{1}\right) \geqslant$ $4 / 9 H$, then we have

$$
r=\frac{H\left(L_{1}\right)+H}{H\left(L_{1}\right)}=1+\frac{H}{H\left(L_{1}\right)} \leqslant 1+\frac{9}{4}=\frac{13}{4} .
$$

If $H\left(L_{1}\right) \leqslant H\left(L_{1}\right) / 4+H / 3$, which means that $H\left(L_{1}\right) \leqslant$ $4 / 9 H$, then

$$
r=\frac{H\left(L_{1}\right)+H}{\frac{H\left(L_{1}\right)}{4}+\frac{H}{3}}
$$

holds. In this case, $r$ is a strictly monotonically increasing function of $H\left(L_{1}\right)$, thus the maximum is attained for $H\left(L_{1}\right)=4 / 9 H$, and $13 / 4$ is the corresponding maximum value. In total, we have $r \leqslant 13 / 4$. Rearrangement of the inequalities above yields

$$
\begin{aligned}
H(L) & \stackrel{(7)}{\leqslant} H\left(L_{1}\right)+H+4 Z \\
& \stackrel{(9)}{=} r \max \left\{H\left(L_{1}\right), \frac{H\left(L_{1}\right)}{4}+\frac{H}{3}\right\}+4 Z \\
& \stackrel{(8)}{\leqslant} \frac{13}{4} O P T(L)+4 Z
\end{aligned}
$$

which proves the claim.

Since $O P T \geqslant Z$ we easily derive the following corollary.

Corollary 10. The absolute approximation ratio of the given algorithm is at most $29 / 4$.

\section{Enumerations and Shifting Technique}

Algorithms B and D generate cutting areas in the strip, resulting in subsets that have to be repacked. We permit further loss of profit by removing more boxes to discard inconvenient layers; the loss will be suitably bounded. The improvement will be at the cost of a considerably larger running time due to a large enumeration; we thus omit a run time analysis. First we introduce a shifting technique to remove sets intersecting the cutting areas and the additional layers. In the sequel we use the notion of a gap which is a rectangular region in the strip that does not intersect the interiors of packed boxes.

Lemma 11. Let $L=\left\{R_{1}, \ldots, R_{n}\right\}$ be a list of boxes with $z_{i} \leqslant \epsilon$ for each $R_{i} \in L$. Suppose $L$ admits a packing into a strip of height at most $h$ and let $m$ be a positive integer. Then we can create $m$ gaps of shape $[0,1] \times[0,1] \times[0, \epsilon]$ in a packing of height $h$ by deleting boxes such that for the remaining list $L^{\prime} \subseteq L$ the inequality $P\left(L^{\prime}\right) \geqslant(1-2(m+1) \epsilon / h) P(L)$ holds. The construction can be done in time polynomial in $n$.

Proof. Consider the original packing in a strip of height exactly $h$. We partition the strip into regions 
of height $\epsilon$ and eventually one region of lower height. More precisely we define $p:=\lceil h / \epsilon\rceil$ and partition the strip of height $h$ into $p$ regions $S_{1}, \ldots, S_{p}$ of shape

$$
[0,1] \times[0,1] \times[0, \epsilon]
$$

where the uppermost region is possibly of lower height. Then for each $i \in\{1, \ldots, p\}$ let $T_{i}=\left\{R_{j} \in L \mid R_{j} \cap S_{i} \neq\right.$ $\emptyset\}$ and let $U_{1}, \ldots, U_{m+1}$ be the $m+1$ sets out of $T_{1}, \ldots, T_{p}$ which have the lowest profit. It is easy to see that removing these from the packing causes a loss of profit which is at most $2(m+1) / p P(L)$ since each box is included in at most two of the sets $T_{1}, \ldots, T_{p}$. We remove $m+1$ sets, since the uppermost region can be among the sets with the lowest profit, but this region might have height lower than $\epsilon$; by removing $m+1$ sets we assert that we create at least $m$ gaps of height $\epsilon$. Let $L^{\prime}$ be the set of remaining boxes; then

$$
\begin{aligned}
P\left(L^{\prime}\right) & \geqslant P(L)-\frac{2(m+1)}{p} P(L)=\left(1-\frac{2(m+1)}{p}\right) P(L) \\
& \geqslant\left(1-2(m+1) \frac{\epsilon}{h}\right) P(L)
\end{aligned}
$$

holds.

Note that the construction above can be carried out in any directions.

Theorem 12. Let $R_{1}, \ldots, R_{n}$ be a list of boxes with $z_{i} \leqslant \epsilon$ for each $R_{i} \in L$ and $V(L) \leqslant \alpha \leqslant 1$. Then it is possible to select $L^{\prime \prime} \subseteq L$ such that $P\left(L^{\prime \prime}\right) \geqslant$ $(1-12 \epsilon) P(L)$ holds, and $L^{\prime \prime}$ admits a feasible packing into at most $\lceil 4 \alpha\rceil$ bins. The construction can be carried out in time polynomial in $n$.

Proof. W.l.o.g., $\epsilon \leqslant 1 / 12$ holds, since otherwise $L^{\prime \prime}=\emptyset$ will do. Use Algorithm A to pack $L$ into a strip which is of height $h \leqslant 4 \alpha$ and two additional layers $L_{1}$ and $L_{2}$ by Theorem 2 . If $h \leqslant 1-2 \epsilon$ we can clearly pack $L$ into 1 bin without losing any profit. For $1-2 \epsilon<h<1$, use Lemma 11 to generate 2 gaps in the strip, which causes a loss of profit of at most $6 \epsilon / h P(L) \leqslant 12 \epsilon P(L)$, since $\epsilon \leqslant 1 / 12$. Moreover, placing $L_{1}$ and $L_{2}$ into the gaps gives a feasible packing into 1 bin. For the remainder of the proof we thus assume $h \geqslant 1$. The following construction is illustrated in Fig.5. Use Lemma 11 to generate at most 5 suitable gaps in the strip, resulting in a loss of profit of at most $12 \epsilon / h P(L)$; since $h \geqslant 1$, this loss is bounded by $12 \epsilon P(L)$. The remaining set of boxes in the strip and $L_{1}$ and $L_{2}$ is denoted as $L^{\prime \prime}$. Consider the 3 cutting unit squares $[0,1] \times[0,1] \times\{i\}$ for $i \in\{1,2,3\}$, and let $L_{3}, L_{4}$ and $L_{5}$ be the sets of boxes in the strip that intersect with these unit squares, respectively. W.l.o.g., none of the sets $L_{1}, \ldots, L_{5}$ is empty; otherwise it is removed from consideration. Note that each of the sets
$L_{1}, \ldots, L_{5}$ can be arranged on a layer of height at most $\epsilon$, so we generate a feasible packing by arranging them into the 5 gaps. In the resulting packing, the 3 cutting unit squares $[0,1] \times[0,1] \times\{i\}$ for $i \in\{1,2,3\}$ do not intersect with any box. Furthermore, all layers $L_{1}, \ldots, L_{5}$ are merged in the strip; the packing can be rearranged into $\lceil 4 \alpha\rceil$ bins.

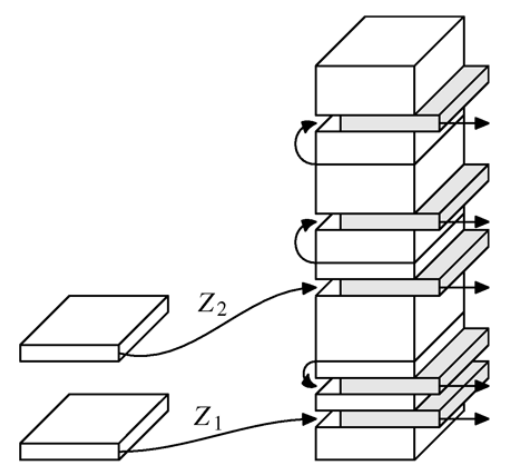

Fig.5. Shifting technique described in Theorem 12.

Like before for any $d \in\{x, y, z\}$ we call a box $R_{i}$ $d-\epsilon$-big $: \Leftrightarrow d_{i} \in(\epsilon, 1]$ and $d-\epsilon$-small $: \Leftrightarrow d_{i} \in(0, \epsilon]$.

We now give the overall algorithm which is based on a separation into boxes that are $d$ - $\delta$-big in all directions and boxes that are $d$ - $\delta$-small in at least one direction. We explain the details in the proof only.

Algorithm E

1. Set $\delta:=\epsilon /[37(7+\epsilon)]$, let

$$
\begin{aligned}
L_{1}:= & \left\{R_{i} \mid R_{i} \text { is } d \text { - } \delta\right. \text {-big } \\
& \text { for each } d \in\{x, y, z\}\}
\end{aligned}
$$

and $L_{2}:=L \backslash L_{1}$.

2. For each $L_{3} \subseteq L_{1}$ such that $\left|L_{3}\right| \leqslant\left\lfloor 1 / \delta^{3}\right\rfloor$ uses an exact algorithm to test the packability of $L_{3}$. Store a feasible $L_{3}$ of maximum total profit.

3. Use an FPTAS for classical KP from [21, 27] to select $L_{4} \subseteq L_{2}$ such that $V\left(L_{4}\right) \leqslant 1$, and $P\left(L_{4}\right) \geqslant$ $(1-\delta) O P T$ holds.

4. Use the construction described below to select $L_{5} \subseteq$ $L_{4}$ which can be packed into at most 6 bins at a small loss of profit.

5. Out of the at most 7 sets generated in Step 2, and Step 4 returns one with the maximum profit.

Theorem 13. Algorithm $E$ is a $(7+\epsilon)$-approximation algorithm for $O K P-3$.

Proof. Note that $\left\lfloor 1 / \delta^{3}\right\rfloor$ is an upper bound for the number of boxes from $L_{1}$ in a feasible solution since $\delta^{3}$ is a lower bound for the volume of each $R_{i} \in L_{1}$. Step 2 can be carried out in time polynomial in $\delta$ and thus polynomial in $1 / \epsilon$ using an exact optimization algorithm as in [18]. We show that in Step 4 at most 6 sets 
are generated, resulting in at most 7 bins in total. Partition $L_{4}$ into 3 subsets $X^{\prime}, Y^{\prime}$ and $Z^{\prime}$ such that in each of these all boxes $R_{i}$ are $d$ - $\delta$-small for the corresponding direction; note that $V\left(X^{\prime}\right)+V\left(Y^{\prime}\right)+V\left(Z^{\prime}\right) \leqslant 1$ holds. We apply the construction from Theorem 12 in each of the three directions. Study the following cases, where $V\left(X^{\prime}\right) \geqslant V\left(Y^{\prime}\right) \geqslant V\left(Z^{\prime}\right)$ holds w.l.o.g.

Case 1. $V\left(X^{\prime}\right) \in(3 / 4,1]$. The boxes in $X^{\prime}$ can be packed into at most 4 bins. We have $V\left(Y^{\prime}\right)+V\left(Z^{\prime}\right)<$ $1 / 4$. This means $V\left(Y^{\prime}\right)<1 / 4$ and $V\left(Z^{\prime}\right)<1 / 4$ hold. Consequently $Y^{\prime}$ and $Z^{\prime}$ can be packed into at most one bin each, resulting in at most 7 bins.

Case 2. $V\left(X^{\prime}\right) \in(1 / 2,3 / 4]$. The boxes in $X^{\prime}$ can be packed into at most three bins. Furthermore, $V\left(Y^{\prime}\right)+V\left(Z^{\prime}\right)<1 / 2$, which means that $V\left(Y^{\prime}\right)<1 / 2$ holds. Consequently the boxes in $Y^{\prime}$ can be packed into at most 2 bins. Furthermore, $V\left(Z^{\prime}\right)<1 / 4$ holds and finally the boxes in $Z^{\prime}$ can be packed into at most 1 bin; this generates at most 7 bins in total.

Case 3. We have $V\left(X^{\prime}\right) \in[0,1 / 2]$. The boxes in $X^{\prime}$ can be packed into at most two additional bins. Furthermore, $V\left(Y^{\prime}\right) \leqslant 1 / 2$ and $V\left(Z^{\prime}\right) \leqslant 1 / 2$ hold. This means that the boxes in $Y^{\prime}$ and $Z^{\prime}$ can be packed into at most two bins each. In total at most 7 bins are generated.

To prove the approximation ratio we fix an optimal solution $S$, and let $P_{1}^{*}$ be the profit of boxes in $S \cap L_{1}$ and $P_{2}^{*}$ be the profit of boxes in $S \cap L_{2}$. Consequently $P_{1}^{*}+P_{2}^{*}=O P T$ holds. Let $P_{1}$ be the profit of the set that is stored in Step 2 and let $P_{2}$ be the profit of the set that is selected in Step 3. By construction we have $P_{1} \geqslant P_{1}^{*}$ and $P_{2} \geqslant(1-\delta) P_{2}^{*}$. Furthermore, by threefold applications of the construction from Theorem 12 the loss of profit in $P_{2}$ is bounded by $36 \delta P_{2}$; let $P_{2}^{\prime}$ denote the remaining list. The profit of the set returned in Step 5 is at least

$$
\begin{aligned}
\frac{1}{7}\left(P_{1}+P_{2}^{\prime}\right) & \geqslant \frac{1}{7}\left(P_{1}^{*}+(1-\delta)(1-36 \delta) P_{2}^{*}\right) \\
& \geqslant \frac{1}{7}\left(P_{1}^{*}+P_{2}^{*}\right)(1-\delta)(1-36 \delta) \\
& =\frac{1}{7} O P T(1-\delta)(1-36 \delta) \geqslant \frac{1}{(7+\epsilon)} O P T
\end{aligned}
$$

which proves the claimed approximation ratio.

Theorem 14. The bound of $(7+\epsilon)$ for the approximation ratio of Algorithm $E$ is asymptotically tight in the sense that it cannot be improved for $\epsilon$ arbitrary small.

Proof. Let $\epsilon \in(0,1 / 4]$ and $\delta$ be defined as in Step 1 of Algorithm E. Note that $\delta \leqslant 1 / 259$. Set $\gamma:=$ $\max \left\{(12 i)^{-1} \mid i \in \mathbb{N},(12 i)^{-1} \leqslant \delta\right\}$. Let $\alpha \in \mathbb{R}_{+}$such that $(3 / 4-\alpha)(1 / 3+\alpha)>1 / 4,(1 / 3-2 \alpha)(3 / 4+6 \alpha)>$
$1 / 4$ and $2 \alpha \leqslant \gamma$ hold. It is easy to see that such an $\alpha$ exists. Note that $\{1 /(2 \gamma), 1 /(3 \gamma), 1 /(4 \gamma), 1 /(6 \gamma)\} \subseteq \mathbb{N}$. We use the boxes,

$$
\begin{aligned}
& A_{i}:=\left(\frac{1}{2}, \frac{1}{2}+\alpha, \gamma\right), \quad i \in\left\{1, \ldots, \frac{1}{\gamma}\right\}, \\
& A:=\left\{A_{1}, \ldots, A_{\frac{1}{\gamma}}\right\} \text {, } \\
& B_{i}:=\left(\frac{1}{2}, \frac{1}{2}+\alpha, \gamma\right), \quad i \in\left\{1, \ldots, \frac{1}{4 \gamma}-1\right\}, \\
& B:=\left\{B_{1}, \ldots, B_{\frac{1}{4 \gamma}-1}\right\} \text {, } \\
& C_{i}:=\left(\gamma, \frac{1}{2}+\alpha, \frac{1}{2}\right), \quad i \in\left\{1, \ldots, \frac{1}{2 \gamma}\right\}, \\
& C:=\left\{C_{1}, \ldots, C_{\frac{1}{2 \gamma}}\right\} \text {, } \\
& D_{i}:=\left(\gamma, 1, \frac{1}{4}+\alpha\right), \quad i \in\left\{1, \ldots, \frac{1}{2 \gamma}\right\} \text {, } \\
& D:=\left\{D_{1}, \ldots, D_{\frac{1}{2 \gamma}}\right\} \text {, } \\
& E_{i}:=\left(\gamma, \frac{1}{3}+\alpha, \frac{3}{4}-\alpha\right), \quad i \in\left\{1, \ldots, \frac{1}{3 \gamma}\right\}, \\
& E:=\left\{E_{1}, \ldots, E_{\frac{1}{3 \gamma}}\right\} \text {, } \\
& F_{i}:=\left(\frac{1}{3}+\alpha, \gamma, \frac{3}{4}-\alpha\right), \quad i \in\left\{1, \ldots, \frac{1}{6 \gamma}-1\right\}, \\
& F:=\left\{F_{1}, \ldots, F_{\frac{1}{6 \gamma}-1}\right\}, \\
& G_{i}:=\left(\frac{1}{3}+\alpha, \gamma, \frac{3}{4}-\alpha\right), \quad i \in\left\{1, \ldots, \frac{1}{2 \gamma}-1\right\}, \\
& G:=\left\{G_{1}, \ldots, G_{\frac{1}{2 \gamma}-1}\right\} \text {, } \\
& H_{i}:=\left(\frac{1}{3}-2 \alpha, \gamma, \frac{3}{4}+6 \alpha\right), \quad i \in\left\{1, \ldots, \frac{1}{2 \gamma}-1\right\}, \\
& H:=\left\{H_{1}, \ldots, H_{\frac{1}{2 \gamma}-1}\right\} \text {, } \\
& R_{1}:=(\delta+\alpha, \delta+\alpha, \delta+\alpha),
\end{aligned}
$$

to define the list $L$. In the following we will show that $L$ admits a feasible packing into the unit cube. Note that each of the sets (without the box $R_{1}$ ) defined above can be arranged to a rectangular block by stacking them on top of each other in the direction $d$ in which they are $d$ - $\delta$-small, see Fig.6. Furthermore, we note that

$$
\begin{aligned}
|A|= & \frac{1}{\gamma}, b r_{z}\left(A_{i}\right)>\frac{1}{4} \quad \text { for } A_{i} \in A, \sum_{A_{i} \in A} z\left(A_{i}\right)=1, \\
|B|= & \frac{1}{4 \gamma}-1, b r_{z}\left(B_{i}\right)>\frac{1}{4} \quad \text { for } B_{i} \in B, \\
& \sum_{B_{i} \in B} z\left(B_{i}\right)=\frac{1}{4}-\gamma, \\
|C|= & \frac{1}{2 \gamma}, b r_{x}\left(C_{i}\right)>\frac{1}{4} \quad \text { for } C_{i} \in C, \sum_{C_{i} \in C} x\left(C_{i}\right)=\frac{1}{2},
\end{aligned}
$$



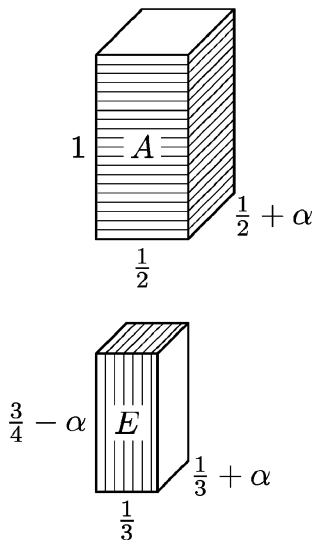
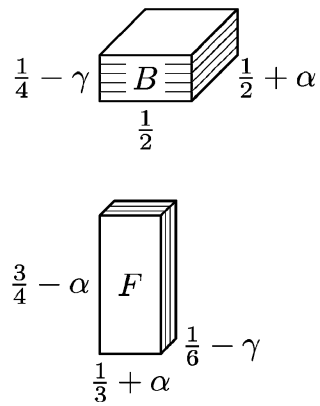
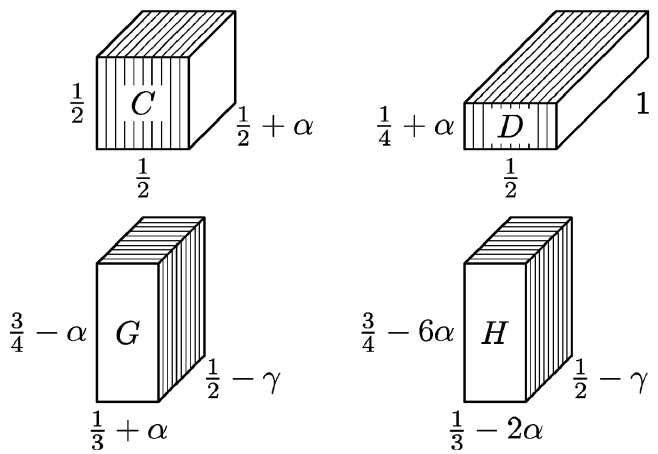

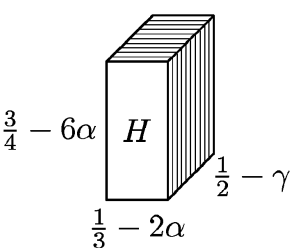

Fig.6. Boxes arranged in blocks.

$$
\begin{aligned}
|D|= & \frac{1}{2 \gamma}, b r_{x}\left(D_{i}\right)>\frac{1}{4} \quad \text { for } D_{i} \in D, \sum_{D_{i} \in D} x\left(D_{i}\right)=\frac{1}{2}, \\
|E|= & \frac{1}{3 \gamma}, b r_{x}\left(E_{i}\right)>\frac{1}{4} \quad \text { for } E_{i} \in E, \sum_{E_{i} \in E} x\left(E_{i}\right)=\frac{1}{3}, \\
|F|= & \frac{1}{6 \gamma}-1, b r_{y}\left(F_{i}\right)>\frac{1}{4} \quad \text { for } F_{i} \in F, \\
& \sum_{F_{i} \in F} y\left(F_{i}\right)=\frac{1}{6}-\gamma, \\
|G|= & \frac{1}{2 \gamma}-1, b r_{y}\left(G_{i}\right)>\frac{1}{4} \quad \text { for } G_{i} \in G, \\
& \sum_{G_{i} \in G} y\left(G_{i}\right)=\frac{1}{2}-\gamma, \\
|H|= & \frac{1}{2 \gamma}-1, b r_{y}\left(H_{i}\right)>\frac{1}{4} \quad \text { for } H_{i} \in H, \\
& \sum_{H_{i} \in H} y\left(H_{i}\right)=\frac{1}{2}-\gamma
\end{aligned}
$$

hold, where $b r_{d}(R)$ denotes the base rectangle in direction $d$ and $d(R)$ denotes component $d$ for a box $R$, and $d \in\{x, y, z\}$. As indicated above, these sets together with the box $R_{1}$ permit a feasible packing since

- $F, E, C$ and $F, E, B$ can be placed next to one another in direction $y$,

- $C, B, D$ can be placed next to one another in direction $z$,

- $A, H$ and $A, G$ and $B, G$ and $C, G$ can be placed next to one another in direction $y$,

which can be verified by elementary calculation, see Fig.7.

In the following we discuss the execution of Algorithm E. The profits necessary for our construction will be described together with the presentation. First we

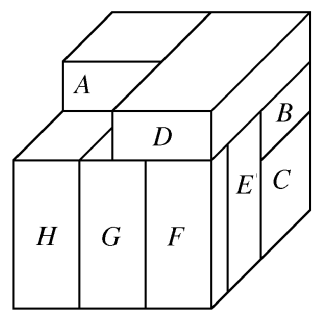

Fig.7. Feasible packing of the boxes into the unit cube.

require $P(L)=1$; this also equals $O P T$ since $L$ permits a feasible packing. In Step 1 we obtain $L_{1}=\left\{R_{1}\right\}$ and $L_{2}=L \backslash\left\{R_{1}\right\}$. Step 2 stores $\left\{R_{1}\right\}$ as a feasible candidate for selection later; here we require $P\left(R_{1}\right)=$ $1 /(7+\epsilon)$. In Step 3 we assume that $L_{4}=L_{2}$ which is possible since $L_{2}$ is an optimal solution for the generated knapsack instance; note that here

$$
P\left(L_{4}\right)=1-1 /(7+\epsilon)=(6+\epsilon) /(7+\epsilon) .
$$

Now we discuss how $L_{4}$ is packed in Step 4 . First $L_{4}$ is partitioned into

$$
X^{\prime}=C \cup D \cup E, \quad Y^{\prime}=F \cup G \cup H, \quad Z^{\prime}=A \cup B .
$$

Then each of these three sets is packed into a strip in the corresponding direction. Note that

$$
\begin{array}{ll}
b r_{x}(R)>\frac{1}{4} & \text { for } R \in X^{\prime}, \\
b r_{y}(R)>\frac{1}{4} & \text { for } R \in Y^{\prime}, \\
b r_{z}(R)>\frac{1}{4} & \text { for } R \in Z^{\prime}
\end{array}
$$

hold. Hence Algorithm E packs all boxes on top of one another in the corresponding direction; we assume that the boxes in each of the sets are packed in "lexicographical" order. Note that the first layer is separated. We denote the heights of the generated strips for $X^{\prime}, Y^{\prime}$ 
and $Z^{\prime}$ by $h_{X^{\prime}}, h_{Y^{\prime}}$ and $h_{Z^{\prime}}$, respectively, and obtain

$$
h_{X^{\prime}}=\frac{4}{3}-\gamma, \quad h_{Y^{\prime}}=\frac{7}{6}-4 \gamma, \quad h_{Z^{\prime}}=\frac{5}{4}-2 \gamma .
$$

Since $\gamma \leqslant \delta \leqslant 1 / 259$, all of these strips have height more than 1 and will later result in 2 bins. Let $X_{1}^{\prime}$ denote the set of boxes that are in or intersect the region $[0,1] \times[0,1] \times[0,1]$ in the strip and set $X_{2}^{\prime}:=X^{\prime} \backslash X_{1}^{\prime}$. We define $Y_{1}^{\prime}, Y_{2}^{\prime}$ and $Z_{1}^{\prime}, Z_{2}^{\prime}$ in a similar way. Now we require that

$$
\begin{aligned}
P\left(X_{1}^{\prime}\right) & =P\left(X_{2}^{\prime}\right)=P\left(Y_{1}^{\prime}\right)=P\left(Y_{2}^{\prime}\right) \\
& =P\left(Z_{1}^{\prime}\right)=P\left(Z_{2}^{\prime}\right)=\frac{1}{6} \frac{6+\epsilon}{7+\epsilon} .
\end{aligned}
$$

The profit is evenly partitioned among the items in the sets. Algorithm E uses the shifting technique from Lemma 11 to generate feasible packings for $X^{\prime}, Y^{\prime}$ and $Z^{\prime}$, respectively, into 2 bins. Hence three gaps of height $\delta$ are generated into each of the strips causing a small loss of profit; the separated layer and the boxes intersecting the cutting square are merged into each strip. It is easy to see that the shifting technique acts only on $X_{1}^{\prime}, Y_{1}^{\prime}$, and $Z_{1}^{\prime}$, respectively, since the profit on each items here is lower than in the corresponding second sets. Thus no item is swapped between sets by the shifting technique. We obtain 6 bins, each containing subsets of $X_{1}^{\prime}, X_{2}^{\prime}, Y_{1}^{\prime}, Y_{2}^{\prime}, Z_{1}^{\prime}$, and $Z_{2}^{\prime}$.

Since each of the sets holds a profit of $(6+\epsilon) /[6(7+\epsilon)]$, each bin holds a profit less than or equal to $(1+\epsilon / 6) /(7+\epsilon)$ which finishes the proof.

\section{Rotational Case}

Finally, we discuss the application of Algorithm E in two different rotational scenarios. In both scenarios rotations of the boxes are only permitted by $90^{\circ}$ around certain axes. In the first case, which we denote by $z$ oriented $\mathrm{OKP}-3$, rotations are only permitted around the $z$-axis. This setting is motivated by packing fragile goods and has been considered in the strip packing variant in [29-31]. In the second case, which we denote by rotational OKP-3, rotations are permitted around all three axes. Note that for both scenarios $Q=(1,1,1)$ does not hold without loss of generality, but is an explicit assumption. Surprisingly, a better approximation ratio can be obtained easily although implicitly, the search space is dramatically enlarged.

We show how Algorithm E can be revised to yield a better approximation ratio for both scenarios. Step 2 is modified in such a way that the exact packing algorithm takes rotations into account, and as before, here at most 1 bin is generated. The most important part is the modification of Step 4. We separate the description of the two scenarios and start with the $z$-oriented setting. Let $Z$ be the set of boxes $R_{i} \in L_{4}$ that are $z$ - $\delta$-small and $X=L_{4} \backslash Z$. We introduce a preprocessing step in which each $R_{i} \in X$ is rotated in such a way that the side length $x_{i}$ is minimal. Consequently, $x_{i} \leqslant \delta$ holds for each $R_{i} \in X$. Hence, the generation of a strip for direction $y$ can be removed; we build only two strips in directions $x$ and $z$ to which the shifting technique from Theorem 12 is applied. This again causes an additional loss of profit which is bounded, however. Let $i \in\{1, \ldots, 4\}$ such that $(i-1) / 4 \leqslant V(Z) \leqslant i / 4$. Then by Theorem 12, exactly $i$ bins are needed to pack the strip in direction $z$. Furthermore, we get

$$
V(X) \leqslant V\left(L_{4}\right)-V(Z) \leqslant 1-\frac{i-1}{4}=\frac{5-i}{4},
$$

which yields a number of $5-i$ bins for the corresponding strip. In total a number of $1+i+(5-i)=6$ bins are needed to pack all items. We call the resulting approach Algorithm $\mathrm{F}$ and obtain the following result.

Theorem 15. Algorithm $F$ is a $(6+\epsilon)$-approximation algorithm for $z$-oriented OKP-3.

For the rotational OKP-3 we have to continue the approach above. Since rotations around all axes are permitted, all items that are $d$ - $\delta$-small for any direction $d \in\{x, y, z\}$ can be packed into one strip in direction $z$ by orienting them such that they are $z$ - $\delta$-small. Since $V\left(L_{4}\right) \leqslant 1$, application of the construction of Theorem 12 yields at most 4 additional bins. We call the resulting approach Algorithm $\mathrm{G}$ and obtain the following result.

Theorem 16. Algorithm $G$ is a $(5+\epsilon)$-approximation algorithm for rotational OKP-3.

\section{Conclusion}

We contributed approximation algorithms for an NP-hard combinatorial optimization problem, where the runtimes of the simpler algorithms are practical. It is of interest whether here an algorithm with ratio $6+\epsilon$ or less exists. We are interested in a reduction of the running time, especially for Algorithm E. In [32] it was proved that it is NP-complete to decide whether a set of squares can be packed into the unit square. However, it is an open problem whether checking the packability of cubes into the unit cube is NP-complete.

Acknowledgements The authors thank the anonymous referees for valuable comments. Florian Diedrich thanks Denis Naddef for hospitality during the preparation of this article. 


\section{References}

[1] Vazirani V V. Approximation Algorithms, Springer, 2001.

[2] Sleator D D K. A 2.5 times optimal algorithm for packing in two dimensions. Information Processing Letters, 1980, 10(1): $37-40$.

[3] Baker B S, Brown D J, Katseff H P. A 5/4 algorithm for two-dimensional packing. Journal of Algorithms, 1981, 2(4): 348-368.

[4] Schiermeyer I. Reverse-fit: A 2-optimal algorithm for packing rectangles. In Proc. ESA, Utrecht, The Netherlands, Lecture Notes in Computer Science 855, Jan van Leeuwen (ed.), Springer, 1994, pp.290-299.

[5] Steinberg A. A strip-packing algorithm with absolute performance bound 2. SIAM Journal of Computing, 1997, 26(2): 401-409.

[6] Kenyon C, Rémila E. A near-optimal solution to a two dimensional cutting stock problem. Mathematics of Operations Research, 2000, 25(4): 645-656.

[7] Jansen K, R Solis-Oba R. New approximability results for 2dimensional packing. Florian Diedrich et al.: Approximation of 3D Orthogonal Knapsack 29 problems. In Proc. MFCS 2007, Ceský Krumlov, Czech Republic, Ludek Kucera, Antonín Licera (eds)., Lecture Notes in Computer Science, 4708, Springer, 2007, pp.103-114.

[8] Miyazawa F K, Wakabayashi Y. An algorithm for the threedimensional packing problem with asymptotic performance analysis. Algorithmica, 1997, 18(1): 122-144.

[9] Jansen K, Solis-Oba R. An asymptotic approximation algorithm for 3D-strip packing. In Proc. 17th ACM-SIAM Symposium on Discrete Algorithms (SODA), 2006, Miami, Florida, USA, pp.143-152.

[10] Bansal N, Han X, Iwama K, Sviridenko M, Zhang G. Harmonic algorithm for 3-dimensional strip packing problem. In Proc. 18th ACM-SIAM Symposium on Discrete Algorithms (SODA), New Orleans, Louisiana, USA, 2007, pp.1197-1206.

[11] Li K, Cheng K H. On three-dimensional packing. SIAM Journal of Computing, 1990, 19(5): 847-867.

[12] Li K, Cheng K H. Heuristic algorithms for on-line packing in three dimensions. Journal of Algorithms, 1992, 13(4): 589605.

[13] W Fernandez de la Vega, G Lueker. Bin packing can be solved within $1+\epsilon$ in linear time. Combinatorica, 1981, 1(4): 349355.

[14] Li R H, Yue M Y. The proof of $\operatorname{FFD}(L) \leqslant(11 / 9) \mathrm{OPT}(\mathrm{L})+$ (7/9). Chinese Science Bulletin, 1997, 42: 1262-1265.

[15] Dósa G. The tight bound of first fit decreasing bin-packing algorithm is $\operatorname{FFD}(I) \leqslant 11=9 \mathrm{OPT}(I)+6=9$. In Proc. ESCAPE, Hangzhou, China, Bo Chen, Mike Paterson, Guochuan Zhang (eds.), Lecture Notes in Computer Science 4614, Springer, 2007, pp.1-11.

[16] Caprara A. Packing two-dimensional bins in harmony. In Proc. 43rd Symposium on Foundations of Computer Science (FOCS), Pittsburgh, PA, USA, 2005, pp.490-499.

[17] Bansal N, Caprara A, Sviridenko M. Improved approximation algorithms for multidimensional bin packing problems. In Proc. 47th Symposium on Foundations of Computer Science (FOCS), Berkeley, CA, USA, 2006, pp.697-708.

[18] Bansal N, Correa J R, Kenyon C, Sviridenko M. Bin packing in multiple dimensions: Inapproximability results and approximation schemes. Mathematics of Operations Research, 2006, 31(1): 31-49

[19] Diedrich F. Approximative algorithmen für rucksackprobleme [Thesis]. Institut für Informatik und Praktische Mathematik der Christian-Albrechts-Universität zu Kiel, 2004.
[20] Jansen K, Zhang G. Maximizing the total profit of rectangles packed into a rectangle. Algorithmica, 2007, 47(3): 323-342.

[21] Kellerer K, Pferschy U, Pisinger D. Knapsack Problems, Springer, 2004

[22] Martello S, Toth P. Knapsack Problems: Algorithms and Computer Implementations. Wiley, 1990.

[23] Rolf Harren. Approximating the orthogonal knapsack problem for hypercubes. In Proc. ICALP (1), Venice, Italy, Lecture Notes in Computer Science, 4051, Michele Bugliesi, Bart Preneel, Vladimiro Sassone, Ingo Wegener (eds.), 2006 , pp.238-249.

[24] Chlebík M, Chlebíková, J. Inapproximability results for orthogonal rectangle packing problems with rotations. In Proc. 6th Conference on Algorithms and Complexity (CIAC), Rome, Italy, 2006, pp.199-210.

[25] Ye D, Zhang G. Online scheduling of parallel jobs with dependencies on 2-dimensional meshes. In Proc. ISAAC, Kyoto, Japan, Lecture Notes in Computer Science 2906, Toshihide Ibaraki, Naoki Katoh, Hirotaka Ono (eds.), Springer, 2003, pp.329-338.

[26] Feldmann A, Sgall J, Teng S H. Dynamic scheduling on parallel machines. Theoretical Computer Science (Special Issue on Dynamic and On-Line Algorithms), 1994, 130(1): 49-72.

[27] Lawler E. Fast approximation algorithms for knapsack problems. Mathematics of Operations Research, 1979, 4(4): 339356.

[28] Ludwig W, Tiwari P. Scheduling malleable and nonmalleable parallel tasks. In Proc. 5th ACM-SIAM Symposium on Discrete Algorithms (SODA), Arlington, Virginia, USA, 1994, pp.167-176.

[29] L Epstein, R van Stee. This side up! In Proc. WAOA, Bergen, Norway, Lecture Notes in Computer Science 3351, Giuseppe Persiano, Roberto Solis-Ob, (eds.), Springer, 2004, pp.48-60.

[30] L Epstein, $\mathrm{R}$ van Stee. This side up! ACM Transactions on Algorithms, 2006, 2(2): 228-243.

[31] Miyazawa F K, Wakabayashi Y. Approximation algorithms for the orthogonal $z$-oriented three-dimensional packing problem. SIAM Journal of Computing, 1999, 29(3): 1008-1029.

[32] Leung J Y T, Tam T W, Wong C S, Young G H, Chin F Y L. Packing squares into a square. Journal of Parallel and Distributed Computing, 1990, 10(3): 271-275.

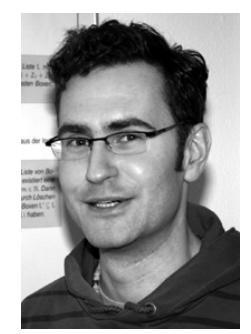

Florian Diedrich received his Diploma in computer science in 2004 from the University of Kiel, Germany, where he is currently a Ph.D. candidate. His research interests include scheduling, geometrically constrained packing problems, approximate solvers for linear programs, and approximation algorithms in general.

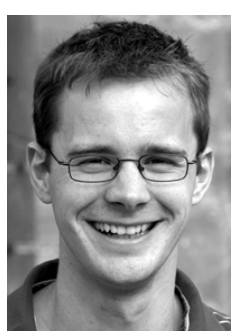

Rolf Harren received his Diploma in computer science from the University of Dortmund in 2007, Germany. He is currently a Ph.D. candidate in computer science at the Max-Planck-Institut für Informatik in Saarbrücken, Germany. His research interests include approximation algorithms and geometrically constrained packing problems. 


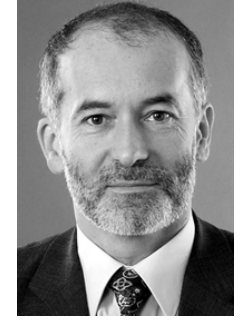

Klaus Jansen received his Ph.D. degree in mathematics in 1990 from the University of Trier, Germany, where he also received his Habilitation in 1994. After temporary professorships in computer science at the University of Duisburg, Germany, and the TU München, Germany, he worked as a researcher at the Max-Planck-Institut für Informatik in Saarbrücken, Germany, and the Istituto Dalle Molle di Studi sull'Intelligenza Artificiale, Lugano, Switzerland. He became an associate professor in computer science in 1999 at the University of Kiel, Germany. His research interests include scheduling, approximation algorithms, linear programming, geometrically constrained packing problems, and complexity theory.

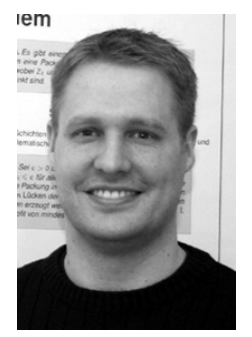

Ralf Thöle received his Diploma in computer science in 2004 from the University of Kiel, Germany, where he is currently a Ph.D. candidate. His research interests include scheduling, geometrically constrained packing problems, hypergraph coloring, graph algorithms, and approximation algorithms in general.

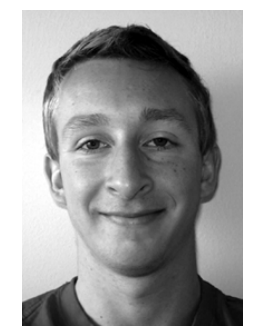

Henning Thomas received his Bachelor's degree in computer science in 2006 from the University of Kiel, Germany, and a Master's degree in computer science in 2008 from the ETH Zurich, Switzerland, where he will start his Ph.D. programme in Novermber 2008. His research interests include combinatorial problems, approximation algorithms, and geometrically constrained packing problems. 